Bull. Austral. Math. Soc.

$53 \mathrm{c} 15,53 \mathrm{c} 20$

VoL. 46 (1992) [177-178]

\title{
ISOMETRIC IMMERSION OF A COMPACT RIEMANNIAN MANIFOLD INTO A EUCLIDEAN SPACE
}

\author{
ShaRIEF Deshmukh
}

\begin{abstract}
We show that an isometric immersion of an $n$-dimensional compact Riemannian manifold of non-negative Ricci curvature with scalar curvature always less than $n(n-1) \lambda^{-2}$ into a Euclidean space of dimension $n+1$ can never be contained in a ball of radius $\lambda$.
\end{abstract}

Jacobowitz [2] proved that an isometric immersion of an $n$-dimensional compact Riemannian manifold with sectional curvature always less than some constant $\lambda^{-2}$ into Euclidean space of dimension $2 n-1$ can never be contained in a ball of radius $\lambda$. This result generalised the results of Tompkins [4], Chern and Kuiper [1] and Otsuki [3]. However it is not known whether such a nonimmersibility theorem holds with the condition on sectional curvature replaced by a suitable condition on Ricci curvature or the scalar curvature. In this note we prove the following co-dimension one nonimmersibility theorem.

TheOREM. Let $E$ be Euclidean space of dimension $n+1$ and $M$ be a compact $n$-dimensional Riemannian manifold of non-negative Ricci curvature whose scalar curvature is less than some constant $n(n-1) \lambda^{-2}$. Then no isometric immersion of $M$ into $E$ is contained in a ball of radius $\lambda$.

Proof: Assume that $\psi: M \rightarrow E$ is the isometric immersion such that $\psi(M)$ is contained in a ball of radius $\lambda$. Thus we can assume that $\|\psi\| \leqslant \lambda$, where $\|$,$\| is the$ norm on $E$ with respect to the Euclidean metric $\langle$,$\rangle . Let N$ be the unit normal vector field to $M$. Then the support function $\rho: M \rightarrow R$ is defined by $\rho=\langle\psi, N\rangle$, and we have the Minkowski's formula

$$
\int_{M}(1+\rho \alpha) d v=0
$$

where $\alpha$ is the mean curvature of $M$.

Denote by $g, \nabla$ and $A$ the Riemannian metric, the Riemannian connection and the Weingarten map on $M$ respectively. The position vector field $\psi$ can be expressed

Received 16 August 1991

Copyright Clearance Centre, Inc. Serial-fee code: 0004-9729/92 \$A2.00+0.00. 
as $\psi=t+\rho N$, where $t$ is a vector field on $M$. Then using Gauss and Weingarten formulae we immediately get

$$
\nabla_{X} t=X+\rho A X
$$

for any vector field $X$ on $M$.

If $\eta$ is a 1 -form dual to $t$, then using equation (2), we obtain

$$
d \eta=0, \quad \delta \eta=n(1+\rho \alpha) .
$$

Also, we obtain

$$
\|\nabla t\|^{2}=n+2 \rho n \alpha+\rho^{2} \operatorname{tr} A^{2} .
$$

Now using $\|t\|^{2}+\rho^{2}=\|\psi\|^{2}$ and the expression for the scalar curvature $S, S=n^{2} \alpha^{2}-$ $\operatorname{tr} A^{2}$, we get

$$
\|\nabla t\|^{2}-(\delta \eta)^{2}=\|t\|^{2} S+n(n-1)-\|\psi\|^{2} S-2 n(n-1)(1+\rho \alpha) .
$$

For a compact Riemannian manifold the following integral formula is known (see $[5]$, p.41)

$$
\int_{M}\left\{\operatorname{Ric}(t, t)-\frac{1}{2}\|d \eta\|^{2}+\|\nabla t\|^{2}-(\delta \eta)^{2}\right\} d v=0
$$

where Ric is the Ricci tensor of $M$.

Using (1), (3) and (4) in the above integral formula we get

$$
\int_{M}\left\{\operatorname{Ric}(t, t)+\|t\|^{2} S+\left(n(n-1)-\|\psi\|^{2} S\right)\right\} d v=0 .
$$

From the hypothesis of the Theorem it follows that $\operatorname{Ric}(t, t) \geqslant 0,\|t\|^{2} S \geqslant 0$ and $\|\psi\|^{2} S \leqslant \lambda^{2} S<n(n-1)$ which contradicts (5). This proves the Theorem.

\section{REFERENCES}

[1] S.S. Chern and N.H. Kuiper, 'Some theorems on the isometric embedding of compact Riemannian manifolds in Euclidean space', Ann. of Math. 56 (1952), 442-430.

[2] H. Jacobowitz, 'Isometric embedding of a compact Riemannian manifold into Euclidean space', Proc. Amer. Math. Soc. 40 (1973), 245-246.

[3] T. Otsuki, 'On the existence of solutions of a system of quadratic equations and its geometrical application', Proc. Japan. Acad. 29 (1953), 99-100.

[4] C. Tompkins, 'Isometric embedding of flat manifolds in Euclidean space', Duke Math. J. 5 (1939), 58-61.

[5] K. Yano, Integral formulas in Riemannian geometry (Marcel Dekker, New York, 1970).

Department of Mathematics

King Saud University

PO Box 2455

Riyadh 11451

Saudi Arabia 